\title{
Evaluation of a symptom-triggered front-loading detoxification technique for alcohol dependence: a pilot study
}

\section{AIMS AND METHODS}

A pilot study was set up to compare a symptom-triggered 'front-loading' detoxification technique with the usual fixed dosage method. A group of 23 in-patients with alcohol dependence were randomised to receive either the intervention technique using diazepam or the standard chlordiazepoxide taper over 10 days.

\section{RESULTS}

The intervention group received a mean dosage of $74 \mathrm{mg}$ diazepam (equivalent to $222 \mathrm{mg}$ chlordiazepoxide) compared with $700 \mathrm{mg}$ chlordiazepoxide in those receiving usual treatment. There was no statistical difference in the severity of alcohol withdrawal symptoms in the two groups, and the intervention group were slightly more satisfied with their treatment than the group undergoing the usual detoxification treatment. Feedback from the nursing staff was positive towards the new approach but highlighted some potential problems for its wider implementation.

\author{
CLINICAL IMPLICATIONS \\ It was possible to use a simple \\ randomised trial design to introduce \\ a new technique for alcohol \\ detoxification to a specialist \\ unit. Symptom-triggered \\ front-loading detoxification \\ using diazepam was as effective \\ as a standard taper technique \\ in terms of withdrawal severity \\ reduction, and was acceptable \\ to both patients and staff. This \\ is potentially a useful \\ technique for busy acute \\ psychiatric wards.
}

The abrupt cessation of alcohol intake in dependent individuals results in a withdrawal syndrome. In some cases this may develop into the more serious consequence of delirium tremens, but this is now uncommon owing to the widespread use of benzodiazepines to attenuate the severity of the withdrawal symptoms (Mayo-Smith, 1997; Williams \& McBride, 1998). Research from the USA, combined with anecdotal evidence in the UK, suggests that the most common method of administering benzodiazepines is by a fixed dosage schedule, with or without additional medication on demand (prn) (Saitz et al, 1995). This means that the dosage of medication used and the rate of reduction are determined before the detoxification process starts. However, because the severity of alcohol withdrawal varies between individuals, these regimens often do not allow individualisation of treatment. Some patients may not receive sufficient medication to prevent withdrawal symptoms, thus putting them at risk of stopping their detoxification programme or experiencing seizures. Others may be at risk of oversedation, and prescribing medication where it is not needed runs the risk of creating a benzodiazepine dependence syndrome in a vulnerable population.

A further disadvantage of fixed dosage regimens is the length of time that they take. In cases in which the expected severity of alcohol withdrawal symptoms merits admission to hospital, it is not uncommon for the medication taper to take 5-10 days. An alternative is the use of a 'front-loading' approach, whereby diazepam is administered at intervals of 1-2 hours until the patient is asymptomatic (Heinala et al, 1990). Such an approach is refined by using a symptom severity rating scale such as the Clinical Institute Withdrawal Assessment for Alcohol - Revised version (CIWA-Ar; Sullivan et al,
1989) to assess the severity of withdrawal symptoms, the results of which dictate the amount of benzodiazepine prescribed. Such regimens may counter many of the problems with traditional fixed dosage techniques highlighted above, with the added bonus of standardised regular monitoring of patients. Furthermore, a number of studies suggest that front-loading regimens use less medication and have a shorter duration, as well as reinforcing the fact that many cases of alcohol withdrawal can be managed without medication at all (Sellers et al, 1983; Wartenberg et al, 1990; Saitz et al, 1994; Wasilewski et al, 1996; Silpakit et al, 1999; Reoux \& Miller, 2000).

Although front-loading detoxification techniques have been reported throughout the world, no clinical trial evidence could be found from a UK centre. There are few reports of randomised controlled trials, and most studies have involved only male patients. Furthermore, it has been usual to exclude patients with a past history of seizures, even though there is other evidence to suggest that this technique is safe in this patient group (Devenyi \& Harrison, 1985). This is significant in the in-patient setting, as a past history of alcohol withdrawal seizures is often a reason for choosing a hospital rather than a home detoxification process. Few of the studies reported any evaluation of patient or staff views about the process.

As a way of introducing this technique in a unit that had previously only ever used fixed dosage chlordiazepoxide schedules without symptom monitoring, we devised a randomised controlled trial to compare the new technique with standard practice. It was decided to use diazepam because of its long half-life, and so the use of a symptom-triggered regimen within the first 24 hours of the detoxification led to therapeutic concentrations of 
the drug being maintained for over 72 hours. Chlordiazepoxide also has a long half-life, but a regimen using diazepam had previously been shown to be safe and

original papers effective by another UK centre (Williams, 2001).

\section{Method}

The Church Road in-patient unit in Birmingham is a 12bedded substance misuse treatment service with resident psychiatric nursing staff and 24-hour specialist medical cover. Half of the beds are dedicated to alcohol detoxification, and admission requires at least one of the following five inclusion criteria:

- a history of seizures related to alcohol withdrawal;

- a history of delirium tremens;

- more than three previous unsuccessful attempts at home detoxification in the past;

- physical or psychiatric comorbidity, making community detoxification unsafe;

- lack of social support for a community detoxification.

All patients meeting ICD-10 criteria for alcohol dependence (World Health Organization, 1992) and requiring inpatient detoxification were considered for randomisation during the 4-month pilot phase. People dependent on substances other than alcohol, or with current severe liver impairment or other major physical illness were excluded, and those unable or unwilling to give informed consent to enter the study were given treatment as usual. All patients attended a pre-admission group meeting during which the study was explained to them and an information leaflet was distributed. On arrival at the unit all patients were assessed with the CIWA-Ar and were invited to join the study. If the patient gave informed consent the researcher conducted a short structured interview and administered the Severity of Alcohol Dependence Questionnaire (SADQ; Stockwell et $a l, 1983)$. The researcher then telephoned a remote randomisation service and the patient was allocated to either symptom-triggered front-loading detoxification or usual treatment. The admitting doctor then completed a full medical assessment, physical examination and blood tests. The study was approved by the local research ethics committee.

The main outcome measures were the amount of medication used in each treatment group, the duration of the detoxification period (defined as the time between the administration of the first and last medication) and the development of any adverse events. Other outcomes of interest were the level of patient satisfaction with symptom control during the detoxification period, and the views of the nursing staff administering the medication. All data analysis was carried out using the Statistical Package for the Social Sciences, version 10.0. Continuous variables that were normally distributed were compared using a $t$-test, and non-parametric continuous data were analysed using the Mann-Whitney U-test.

\section{Intervention}

All qualified nursing staff working on the in-patient unit attended a training session on how to use the CIWA-Ar, at which an introductory lecture was followed by a series of video recordings of patients at various stages of alcohol withdrawal. The staff were asked to rate these cases using the CIWA-Ar, and the results were discussed and compared with a gold standard rating. The staff then practised rating patients admitted to the unit prior to the start of the trial, and the results were discussed during the weekly ward review.

Participants in the intervention group were assessed every 90 minutes using the CIWA-Ar, with each assessment taking approximately 10 minutes. Those scoring 11 or more received $20 \mathrm{mg}$ of diazepam, whereas those with a score of 10 or less received no medication. The process was discontinued when the patient scored 10 or less on two consecutive occasions, after which no further medication was administered. Participants in the control group received $30 \mathrm{mg}$ of chlordiazepoxide every 6 hours on the first day, with the dose tapering to zero according to a defined regimen over a 10 -day period. Nursing staff were also able to dispense up to an additional $20 \mathrm{mg}$ of chlordiazepoxide every 6 hours if they judged that the patient required it. The CIWA-Ar was used to assess all patients twice daily prior to the administration of medication for the first 10 days of the period of admission.

\section{Results}

The pilot phase ran for 4 months, and during this time 39 patients were admitted to the unit for alcohol detoxification. A total of 16 patients ( 10 men, 6 women) were excluded from the study: 5 were unable or unwilling to give informed consent to take part in the trial; 4 had severe current mental health problems; 3 had alcoholrelated liver disease; 2 had completed their detoxification prior to admission; 1 was dependent on cocaine; and 1 was excluded owing to the lack of a trained CIWA-Ar rater at the time of admission.

\section{Detoxification process}

A total of 23 participants were randomised: 11 to the intervention and 12 to treatment as usual. The characteristics of the two groups at baseline assessment are shown in Table 1, with the gender mix the only difference reaching statistical significance. Nine of the sample had well-documented evidence of previous seizures related to alcohol withdrawal, and the mean SADQ score of the whole sample (40.4) suggested severe alcohol dependence (Stockwell et al, 1983). All 23 participants completed the full detoxification process, and no one left the unit prematurely. The intervention group received a mean dose of $74 \mathrm{mg}$ of diazepam (equivalent to $222 \mathrm{mg}$ chlordiazepoxide; Bazire, 2000), compared with $700 \mathrm{mg}$ of chlordiazepoxide in the control group $(P<0.001)$. The mean length of the detoxification period (as defined by administration of medication) was 8.2 hours, compared with 242 hours in the control group $(P<0.001)$. However, 
Table 1. Characteristics of the two treatment groups at baseline assessment

\begin{tabular}{|c|c|c|}
\hline & $\begin{array}{l}\text { Fixed dosage } \\
\text { detoxification } \\
\quad(n=12)\end{array}$ & $\begin{array}{c}\text { STFL } \\
\text { detoxification } \\
(n=11)\end{array}$ \\
\hline Age, years: mean (s.d.) & $44.1 \quad(6.1)$ & $46.4 \quad(9.6)$ \\
\hline \multicolumn{3}{|l|}{ Gender, $n$} \\
\hline Male & 9 & 5 \\
\hline Female & 3 & 6 \\
\hline $\begin{array}{l}\text { Duration of heavy } \\
\text { alcohol use, years: } \\
\text { mean (s.d.) }\end{array}$ & $13.2 \quad(8.3)$ & $11.1 \quad(7.2)$ \\
\hline $\begin{array}{l}\text { Number of days person } \\
\text { drank alcohol in last } 30 \\
\text { days, n: mean (s.d.) }\end{array}$ & $28.3 \quad(5.8)$ & $30 \quad(0)$ \\
\hline $\begin{array}{l}\text { Time since last alcoholic } \\
\text { drink, h: mean (s.d.) }\end{array}$ & $5.5 \quad(4.9)$ & $5.2 \quad(5.2)$ \\
\hline $\begin{array}{l}\text { Units of alcohol per } \\
\text { day, n: mean (s.d.) }\end{array}$ & $32.7(23.5)$ & $29.3(13.5)$ \\
\hline $\begin{array}{l}\text { SADQ score on } \\
\text { admission: mean (s.d.) }\end{array}$ & $37.8(14.9)$ & $43.3 \quad(9.9)$ \\
\hline $\begin{array}{l}\text { Previous episodes of } \\
\text { medically supported } \\
\text { alcohol detoxification, } \\
n \text { : mean }\end{array}$ & 1.8 & 1.7 \\
\hline
\end{tabular}

SADQ, Severity of Alcohol Dependence Questionnaire; STFL, symptomtriggered front-loading detoxification.

despite these differences, there was little difference in the severity of alcohol withdrawal between the two groups throughout the 10 days studied (Fig. 1). Comparison of the two groups at each time point using a MannWhitney $U$-test confirmed the null hypothesis.
Only one patient in the study had any significant complications during the detoxification: this was a 39year-old woman in the intervention group who was observed to have a generalised tonic-clonic seizure during the evening of the first day of admission to the unit. At this point the level of severity of the patient's withdrawal symptoms had been measured every 90 minutes and no symptom of alcohol withdrawal had been noted (CIWA-Ar score <5). On more detailed questioning the patient admitted to a history of heavy zopiclone use which had stopped abruptly 2 days prior to admission, and furthermore she had experienced epileptic seizures during periods of abstinence from alcohol in the past.

\section{Patient satisfaction}

At the end of the detoxification period (10 days after admission to the unit) participants were asked to complete a Patient Satisfaction Questionnaire anonymously. The participants rated the number of days out of the past 10 that they had been troubled by 10 different withdrawal symptoms, giving a maximum score of 100 . The intervention group reported a lower median level of adverse symptoms than did the group receiving the traditional detoxification (14.0 v. 29.5, $P=0.267$ ). In addition, the participants rated their satisfaction with the staff, the medication and the detoxification process as a whole on a scale of 1 to 10. Despite the shorter period of administration of medication in the intervention group, their mean satisfaction rating was slightly higher than that of the control group (28.0 v. 27.3, $P=0.152$ ).

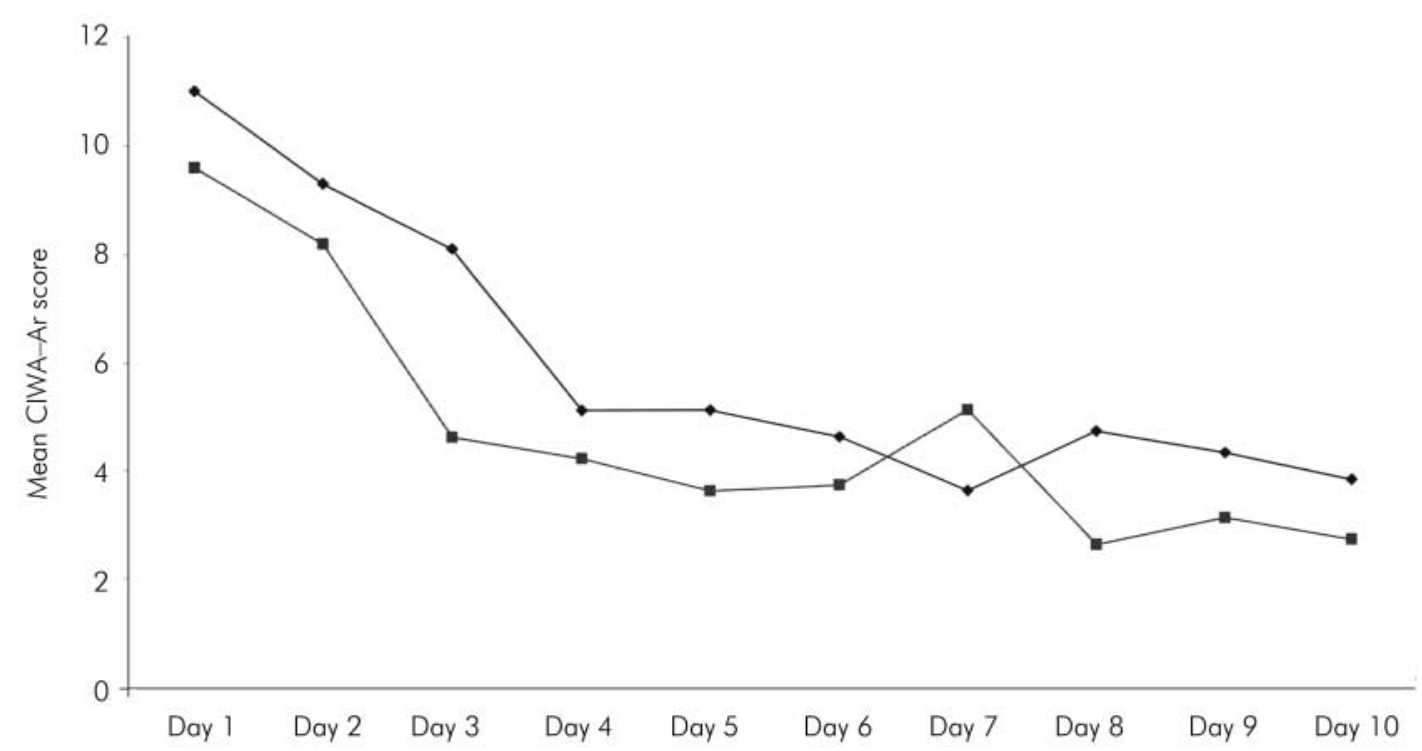

Fig. 1. Mean daily score on the Clinical Institute Withdrawal Assessment for Alcohol - Revised scale (CIWA-Ar) for patients undergoing symptom-triggered front-loading detoxification ( $\mathbf{\square}$ ) compared with the usual fixed dosage regimen 


\section{Nursing staff}

original

papers
Five of the six members of the nursing staff directly involved with the care of patients in the study were interviewed to determine their opinions about the use of symptom-triggered front-loading detoxification and its results. Only one member of staff had been aware of the front-loading detoxification technique prior to the start of the study. All the staff felt that the technique gave the patients better symptom control, and they were positive about the reduced duration of detoxification and the lower doses of medication used. Participants were also noted to be less sedated throughout the detoxification period, and were able to engage in psychological group work at an earlier stage than with the traditional approach. The technique was felt to be empowering for nursing staff, giving them more direct control over the care of their patients. Many of the problems anticipated by the nursing staff did not arise, but a need for ongoing training was highlighted and some problems with low staffing levels during the trial period were also exposed.

\section{Discussion}

This was a pilot study to test the efficacy and feasibility of introducing a new medical treatment of the alcohol withdrawal syndrome, and as such it had a number of methodological limitations, including the need to use different medications in the two treatment groups and the lack of either single or double masking. However, the study clearly demonstrated that the symptom-triggered front-loading detoxification technique resulted in less medication usage and a shorter duration of medical treatment without an increase in objectively measured withdrawal severity or complications. Furthermore, the sample included high proportions of women and of participants with a history of alcohol withdrawal-related seizures. Patients and nursing staff were both satisfied with the new procedure, and the results were so positive that the pilot was terminated after the initial 4-month phase. It is now hoped that by introducing the technique in the form of a clinical trial it will be easier to implement it in everyday practice.

Front-loading detoxification techniques appear to yield potentially significant benefits in terms of bed occupancy. All patients in this study were allowed to complete the full 3-week in-patient period, as this was the duration of admission promised when they were initially placed on the waiting list for treatment. However, the reduced length of the medication period with this technique allows the option of a much shorter admission period, or less cognitive impairment for patients entering the relapse prevention group programme also available in the unit. Such techniques might be well suited to other services such as acute medical units (Lange-Asschenfeldt et al, 2003), where an initial investment in training might be rewarded by shorter durations of admission.

\section{References}

BAZIRE, S. (2000) Psychotropic Drug Directory. Snow Hill: Quay Books.

DEVENYI, P. \& HARRISON, M. L. (1985)

Prevention of alcohol withdrawal

seizures with oral diazepam loading.

Canadian Medical Association Journal, 132, 798-800.

HEINALA, P., PIEPPONEN,T. \&

HEIKKINEN, H. (1990) Diazepam loading in alcohol withdrawal: clinical pharmacokinetics. International Journal of Clinical Pharmacology, Therapy and Toxicology, 28, 211-217.

LANGE-ASSCHENFELDT, C., MULLER, M. J., SZEGEDI, A., et al (2003) Symptom-triggered versus standard chlormethiazole treatment of inpatient alcohol withdrawal: clinical implications from a chart analysis. European Addiction Research, 9, 1-7.

MAYO-SMITH, M. F. (1997)

Pharmacological treatment of alcohol withdrawal: a meta-analysis and evidence-based practice guideline. American Society of Addiction

MedicineWorking Group on

Pharmaceutical Management of

Alcohol Withdrawal. JAMA, 278,

144-151.

REOUX, J. P. \& MILLER, K. (2000) Routine hospital alcohol detoxification practice compared to symptom triggered management with an objective withdrawal scale. American Journal on Addictions, 9, 135-144.

SAITZ, R., MAYO-SMITH, M. F. ROBERTS, M. S., et al (1994) Individualized treatment for alcohol withdrawal: a randomized double-blind controlled trial. JAMA, 272, 519-523.

SAITZ, R., FRIEDMAN, L. S. \& MAYOSMITH, M. F. (1995) Alcohol

withdrawal: a nationwide survey of inpatient treatment practices. Journal of General Internal Medicine, 10, 479-487.

SELLERS, E. M., NARANJO, C. A. HARRISON, M., et al (1983) Diazepam loading: simplified treatment of alcohol

withdrawal. Clinical Pharmacology and Therapeutics, 34 , 822-826.

SILPAKIT, C., SILPAKIT, O. \& KUMYAN, S. (1999) Treatment of alcohol withdrawal: a fixed schedule regimen versus symptom-triggered regimen International Medical Journal, $\mathbf{6}$, 287-289.

STOCKWELL,T., MURPHY, D. \& HODGSON, R. (1983) The Severity of Alcohol Dependence Questionnaire: its use, reliability and validity. British Journal of Addiction, 78, 145-155.

SULLIVAN, J. T., SYKORA, K., SCHNIDERMAN, J., et al (1989)

Assessment of alcohol

withdrawal: the Revised Clinical InstituteWithdrawal Assessment for Alcohol Scale (CIWA-Ar). British Journal of Addiction, 84, 1353-1357.

WARTENBERG, A. A., NIRENBERG,T. D. LIEPMAN, M. R., et al (1990) Detoxification of alcoholics: improving care by symptom-triggered sedation. Alcoholism: Clinical and Experimental Research, 14,71-75.

WASILEWSKI, D. MATSUMOTO, $\mathrm{H}$, KUR, E., et al (1996) Assessment of diazepam loading dose therapy of delirium tremens. Alcohol and Alcoholism, 31, 273-278.

WILLIAMS, S. (2001) Introducing an inpatient treatment for alcohol detoxification into a community setting. Journal of Clinical Nursing, 10 635-642

WILLIAMS, D. \& MCBRIDE, A. J. (1998) The drug treatment of alcohol withdrawal symptoms: a systematic review. Alcohol and Alcoholism, 33, 103-115.

WORLDHEALTHORGANIZATION (1992) The ICD-10 Classification of Mental and Behavioural Disorders. Clinical Description and Diagnostic Guidelines. Geneva: WHO

Ed Day Senior Clinical Lecturer in Addiction Psychiatry, University of Birmingham, Queen Elizabeth Psychiatric Hospital, MindelsohnWay, Edgbaston, Birmingham B15 2QZ (tel: 0121678 2372; fax: 0121678 2351; e-mail: e.j.day@ bham.ac.uk), Jaimain Patel medical student, University of Birmingham Medical School, George Georgiou Consultant Addiction Psychiatrist, Birmingham 